\title{
A highly-underactuated robotic hand with force and joint angle sensors
}

\author{
Long Wang*, Joseph DelPreto**, Sam Bhattacharyya*, Jonathan Weisz***, Peter K. Allen*** \\ $*$ Mechanical Engineering, $* *$ Electrical Engineering, and $* * *$ Computer Science, Columbia University
}

\begin{abstract}
This paper describes a novel underactuated robotic hand design. The hand is highly underactuated as it contains three fingers with three joints each controlled by a single motor. One of the fingers ("thumb") can also be rotated about the base of the hand, yielding a total of two controllable degrees-of-freedom. A key component of the design is the addition of position and tactile sensors which provide precise angle feedback and binary force feedback. Our mechanical design can be analyzed theoretically to predict contact forces as well as hand position given a particular object shape
\end{abstract}

Key words - Robotic Hands - Underactuation - Tactile sensors

\section{INTRODUCTION}

Underactuated robotic hands have become quite popular over the last few years, for a number of reasons. Hands such as $[1,2,3,4]$ occupy a niche among the wide spectrum of robotic hands that lie between simplistic 2-fingered industrial grippers and complex 5-fingered humanoid hands such as the shadow hand [5]. Utilizing usually one actuator or less to curl a single finger, these hands allow a much more simplified control scheme compared to traditional fully actuated multi-finger hands. The joint compliance and multi-fingered configurations in these under-actuated hands also gives them the beneficial property of being able to successfully grasp complex objects of relatively unknown shape and orientation (see [6]) as well as to be easily integrated into larger systems such as an arm to interact with the environment ([7]). Low cost, simple design, and the potential for mass marketability all make under-actuating hands quite promising for current and future development in robotic prostheses and humanoid robotics (for example, see [8]).

An open question relating to these hands is just how much underactuation can be tolerated before the hand loses its ability to grasp and manipulate objects. This paper discusses the design of the Columbia Hand, which is a highly under-actuated hand, containing 3 fingers with 3-DOF each, and only two actuators: one for closing the fingers and one for rotating the thumb around the base. To supplement the lack of controllable DOF's, a rich set of sensors has been included that can estimate 1) the joint angles and 2) tactile contacts on each segment of the hand. While some research has been done regarding the integration of tactile sensors with the principle of underactuation (e.g. $[9,10]$ ), very little work has been done to integrate both position and tactile feedback. Such feedback, combined with the adaptability inherent to underactuation, allows for sophisticated control algorithms and enhanced grasp quality of a wide variety of objects.

This work was supported in part by the National Science Foundation under Grant IIS-090414

\section{PRINCIPLES OF TP-UA Mechanisms}

As shown in Fig. 1, the Tendon-Pin (TP) Underactuated (UA) mechanism was integrated on each joint of each finger. This design integrates small segments of a robotic finger via pin joints and a tendon, allowing for a robust design which is both easily controlled and inherently adaptable to a wide variety of objects. The response of this TP-UA when closing on an object can be divided into three essential stages: the initial stage, the pre-shaping stage, and the closing stage.

\section{A. Initial Stage}

In the initial stage, shown in Fig. 1 (a), the finger is straightened by return springs. There is a tendon going through holes on every segment, with one end fixed to the distal segment and the other connected to the lifting mechanism actuator. As shown in Fig. 1, the grasp process is temporarily considered with only the first two DOFs for convenience.
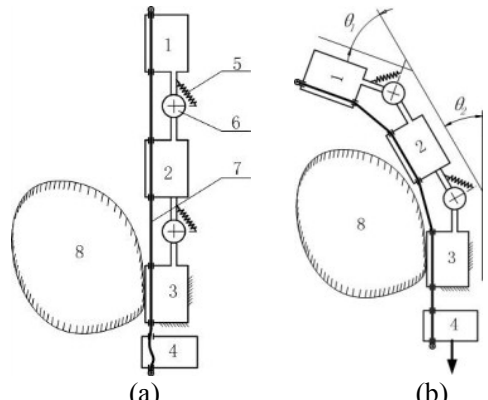

(b)

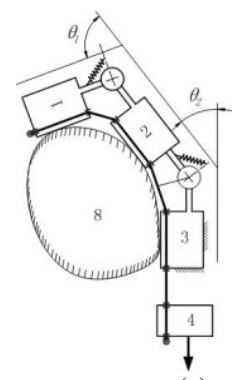

(c)

Fig. 1.The principles of a tendon-pin under-actuated finger, illustrating (a) the initial stage, (b) the pre-shaping stage, and (c) the closing stage. Notes: 1 - the distal segment; 2 - the middle segment; 3 - the fixed segment; 4 - the lifting mechanism; 5 - the return spring; 6 - the joint pin; 7 - the tendon; 8 - the grasped object

\section{B. Pre-Shaping Stage}

The pre-shaping stage will be considered as the interval beginning when actuation is applied and ending when any segment touches the object. During this time, the finger acquires a pre-shaped bend.

As shown in Fig. 1 (b), once the actuator moves down and applies a tensile force on the tendon, the finger will start closing. All the joints will be rotating simultaneously in a coupled relationship. The specifications of this process and coupled relationship depend on design parameters such as tendon position (distance from joint) and the return spring, which will be discussed below.

\section{Closing Stage}

The closing stage will describe the interval beginning with the moment of object contact and ending when no segment 
can continue to move (when the grasp is completed).

As shown in Fig. 1 (c), if the middle segment is blocked and the tendon is continuously pulled down, the distal segment can continue to bend - the two joint angles have been decoupled by the object.

In this way, the TP-UA finger can complete a grasp task with a single actuator. There are several advantages:

1) Small volume and weight due to the simple mechanism, especially compared to link and gear style UA mechanisms;

2) Reduced number of required lifting mechanisms. Specifically, this paper proposes a closing system of 9 DOFs actuated by a single lifting mechanism.

3) Increased anthropomorphism. Due to its mimicry of human muscles, the pre-shaping process of a tendon-based design naturally reflects human movement (for example, see $[11,6])$. This transmission will be discussed in detail below.

\section{FINGER MODEL \& MECHANICAL ANALYSIS}

To effectively apply the TPUA in robotic fingers, a feasible design must be considered with all parameters optimized regarding geometry, mechanics, and motion planning. While the mechanics of a linkage-driven self-adaptive finger is discussed in [12], this analysis demonstrates the mechanics of a tendon-based adaptive underactuated hand. As shown in Fig. 2 and Fig. 4, a mechanical analysis is addressed in detail for each of the three stages introduced above.

\section{A. Assumptions, Parameters, and Symbol Definition}

The symbols used in this section are defined in TABLE I. According to Fig. 2 (a), there are four important design parameters specifying this model, including $L_{0}, L_{l}, R_{0}$, and $K$.

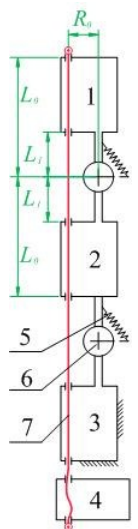

(a)

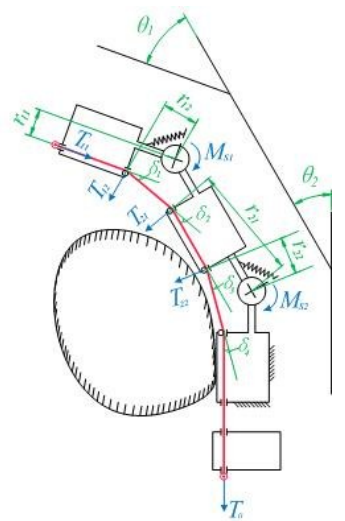

(b)
Fig. 2. The mechanical analysis of the pre-shaping stage

Notes: 1 - the distal segment; 2 - the middle segment; 3 - the fixed segment; 4 - the lifting mechanism; 5 - the return spring; 6 - the joint pin; 7 - the tendon; 8 - the grasped object

There are several assumptions applied to construct this model. First, the finger mechanism itself is assumed to be frictionless; thus, every contact point of the tendon can be modeled as a smooth pulley (shown in Fig. 2 (b)). Note that this model does, however, account for friction between the finger and the object. Second, the finger components are assumed to be massless; thus, there are no gravitational effects as the joints rotate. Third, the model applies symmetric and identical segments. Finally, all finger movements will be considered as quasi-static processes.

TABLE I

Symbol Definition

\begin{tabular}{|c|c|c|}
\hline Symbol & Description & Position \\
\hline$L_{0}$ & The height of each segment. & Fig. 2(a) \\
\hline$L_{1}$ & The height of closer tendon turning. & Fig. 2(a) \\
\hline$R_{0}$ & The width between tendon holes and joints. & Fig. 2(a) \\
\hline K & The stiffness coefficient of return springs. & Fig. 2(a) \\
\hline$\theta_{i}$ & The rotation angle of the $i$ th joint. & Fig. 2(b) \\
\hline$\delta_{i}$ & The $i$ th tendon turning angle. & Fig. 2(b) \\
\hline$T_{0}$ & $\begin{array}{l}\text { The applied force on the tendon produced by the } \\
\text { lifting mechanism. }\end{array}$ & Fig. 2(b) \\
\hline$T_{11}$ & $\begin{array}{l}\text { The force applied on the stop ball produced by } \\
\text { the tendon. }\end{array}$ & Fig. 2(b) \\
\hline $\begin{array}{l}T_{12}, T_{21}, \\
\text { and } T_{22}\end{array}$ & $\begin{array}{l}\text { The force applied on tendon turnings of } \\
\text { segments produced by the tendon. }\end{array}$ & Fig. 2(b) \\
\hline$r_{11}$ & $\begin{array}{l}\text { The lever arm of the moment with respect to the } \\
\text { first joint produced by } T_{11} \text {. }\end{array}$ & Fig. 2(b) \\
\hline $\begin{array}{l}r_{12}, r_{21} \\
\text { and } r_{22}\end{array}$ & $\begin{array}{l}\text { The lever arms of the moment with respect to the } \\
1^{\text {st }} \text { and } 2^{\text {nd }} \text { joint produced by } T_{12}, T_{21} \text { and } T_{22} \text {. }\end{array}$ & Fig. 2(b) \\
\hline$M_{S i}$ & $\begin{array}{l}\text { The torque produced by the return spring, } \\
\text { applied at the } i \text { th joint toward the } i \text { th segment. }\end{array}$ & Fig. 2(b) \\
\hline$F_{i}$ & $\begin{array}{l}\text { The reaction force applied on the } i \text { th segment } \\
\text { produced by the object - this force includes the } \\
\text { effects of friction. }\end{array}$ & Fig. 4 \\
\hline$k_{i}$ & The lever arm of $F_{i}$ & Fig. 4 \\
\hline$n_{i}$ & $\begin{array}{l}\text { The direction normal to the finger surface at the } \\
\text { contact point of the } i \text { th segment. }\end{array}$ & Fig. 4 \\
\hline$\tau_{i}$ & $\begin{array}{l}\text { The direction tangential to the finger surface at } \\
\text { the contact point of the } i \text { th segment; }\end{array}$ & Fig. 4 \\
\hline$N_{i}$ & $\begin{array}{l}\text { The projection of } F_{i} \text { onto the } n_{i} \text { direction, which } \\
\text { equals the normal force produced by the object. }\end{array}$ & Fig. 4 \\
\hline$f_{i}$ & $\begin{array}{l}\text { The projection of } F_{i} \text { onto the } \tau_{i} \text { direction, which } \\
\text { equals the friction produced on contact surfaces. }\end{array}$ & Fig. 4 \\
\hline$\psi_{i}$ & $\begin{array}{l}\text { The angle between } f_{i} \text { and } F_{i} \text {, which indicates the } \\
\text { friction angle (rad); }\end{array}$ & Fig. 4 \\
\hline
\end{tabular}

\section{B. Analysis during the Pre-Shaping Stage}

Fig. 2 (b) shows the mechanical analysis of the pre-shaping stage, during which the joint angles are coupled. The mathematical derivation below investigates the exact nature of this coupling - given the above design parameters, one can determine the unique joint angle coupling process.

The following results can be proved geometrically assuming the symmetric configuration described above:

$$
\begin{gathered}
\delta_{1}=\delta_{2}=\frac{\theta_{1}}{2}, \delta_{3}=\delta_{4}=\frac{\theta_{2}}{2} \\
\left\{\begin{array}{l}
r_{11}=R_{0}, r_{12}=l_{1} \sin \left(\gamma_{1}-\delta_{1} / 2\right) \\
r_{21}=l_{0} \sin \left(\gamma_{0}-\delta_{2} / 2\right), r_{22}=l_{1} \sin \left(\gamma_{1}-\delta_{3} / 2\right)
\end{array}\right.
\end{gathered}
$$

where $l_{i}=\sqrt{L_{i}^{2}+R_{0}^{2}}, \gamma_{i}=\tan ^{-1}\left(L_{i} / R_{0}\right), i=0,1$

But note that if the design is not symmetric, the following are still valid:

$$
\delta_{1}=\eta_{1} \delta_{2}, \text { s.t. } \delta_{1}+\delta_{2}=\theta_{1} ; \delta_{3}=\eta_{2} \delta_{4}, \text { s.t. } \delta_{3}+\delta_{4}=\theta_{2}\left(1^{\prime}\right)
$$

where $\eta_{i}$ can be a design parameter ranging from 0 to 1 .

Second, consider force equilibrium, assuming quasi-static processes. According to the force balance at each tendon turning point, the following results can be derived:

$T_{11}=T_{0}, T_{12}=2 T_{0} \sin \delta_{1}, T_{21}=2 T_{0} \sin \delta_{2}, T_{22}=2 T_{0} \sin \delta_{3}(4)$

Third, consider moment equilibrium, assuming quasi-static processes. According to the moment at each joint, the 
following results can be derived:

$$
\left\{\begin{array}{l}
T_{11} \cdot r_{11}+T_{12} \cdot r_{12}=M_{S 1}, \quad M_{S 1}=M_{S 10}+K \theta_{1} \\
T_{21} \cdot r_{21}+T_{22} \cdot r_{22}=M_{S 2}-M_{S 1}, \quad M_{S 2}=M_{S 20}+K \theta_{2}
\end{array}\right.
$$

$M_{S 10}, \quad M_{S 20}$ are pre-loading torques applied on the corresponding joints (due to stretching of the springs in the initial stage), and $M_{S 1}, M_{S 2}$ are due to additional stretching as the finger rotates.

Summarizing (1) through (5), it can be concluded that

$$
\begin{gathered}
T_{0}=\frac{M_{S 10}+K \theta_{1}}{R_{0}+2 l_{1} \sin \left(\theta_{1} / 2\right) \sin \left(\gamma_{1}-\theta_{1} / 4\right)}=P\left(\theta_{1}\right) \\
\sin \frac{\theta_{2}}{2} \sin \left(\gamma_{1}-\frac{\theta_{2}}{4}\right)-\frac{K}{2 l_{1} P\left(\theta_{1}\right)} \theta_{2}=Q\left(\theta_{1}\right)
\end{gathered}
$$

Thus, given the design parameters, one can calculate the relationship between $\theta_{1}$ and $\theta_{2}$ (Fig. 3b), i.e. how the two angles are coupled, by substituting $\theta_{1}$ into (6) and solving (7). In addition, it is important to note that the tendon force $T_{0}$ (the force the actuator must supply) is a function of only $\theta_{l}$.

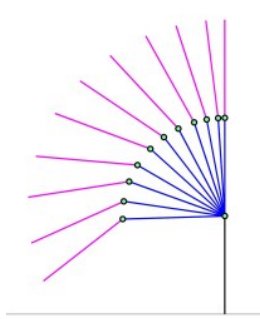

(a) (b)

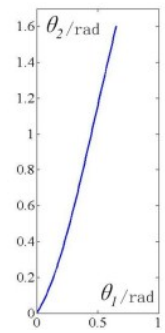

Fig. 3. The relationship between $\theta_{l}$ and $\theta_{2}$, demonstrating the ability to predict the pre-shaping position as well as a strong resemblance to human movement.(a) simulates the pre-shaping while (b) shows how the two angles are coupled. Both analyses are done with the same parameter configuration as the specified design which will be discussed later, and the pre-loading torque is set to zero for convenience.

From Fig. 3, it can be concluded that a natural pre-shaping process similar to a human hand is obtained during this stage.

\section{Analysis during the Closing Stage}

Now consider the geometry and mechanics during the closing stage, as shown in Fig. 4.

Unlike the pre-shaping stage, the two angles $\theta_{1}$ and $\theta_{2}$ are not coupled. Instead, they are now decoupled by the shape and contact force of the grasped object. Thus the relevant investigation of this stage regards the determination of the grasp force given a particular grasped object, i.e. given how $\theta_{1}$ and $\theta_{2}$ are distributed, one can compute the grasp force.

First, note that the geometric issue is the same as in the pre-shaping stage, which means that equations (1), (2), and (3) can still be used in this section.

Second, the force equilibrium is the same as in the pre-shaping stage with the exception of the contact forces, so equation (4) remains valid. The contact forces are the superposition of normal and frictional forces, which depend on the coefficient of friction and the object's shape. Here, the directions are assumed to be known for the particular object.

Third, the moment equilibrium is similar to that of the previous stage. Thus, (5) can be modified to obtain

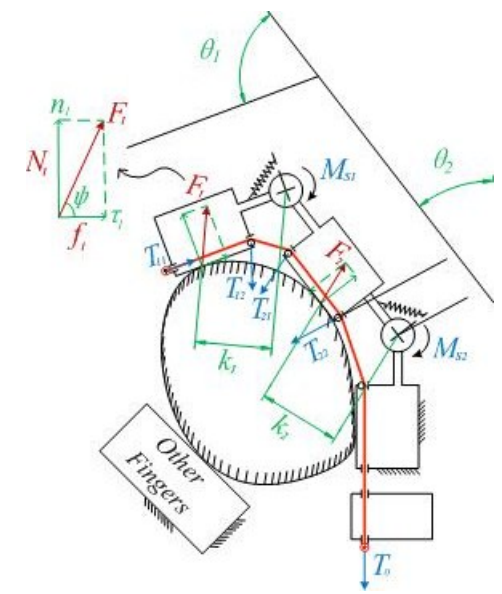

Notes: the labels are the same as those in Fig. 2.

Fig. 4. The mechanical analysis of the closing stage

$$
\left\{\begin{array}{l}
T_{11} \cdot r_{11}+T_{12} \cdot r_{12}-F_{1} \cdot k_{1}=M_{S 1}\left(\theta_{1}\right) \\
T_{21} \cdot r_{21}+T_{22} \cdot r_{22}-F_{2} \cdot k_{2}=M_{S 2}\left(\theta_{2}\right)-M_{S 1}\left(\theta_{1}\right)
\end{array}\right.
$$

which can be rewritten as

$$
\left\{\begin{array}{l}
F_{1}=k_{1}^{-1} A\left(T_{0}, \theta_{1}\right) \\
F_{2}=k_{2}^{-1} B\left(T_{0}, \theta_{1}, \theta_{2}\right)
\end{array} \text { or }\left[\begin{array}{l}
F_{1} \\
F_{2}
\end{array}\right]=\left[\begin{array}{l}
k_{1}^{-1} \\
k_{2}^{-1}
\end{array}\right]^{T} \cdot\left[\begin{array}{ll}
A & 0 \\
0 & B
\end{array}\right]\right.
$$

where the functions $A$ and $B$ can be determined from (8).

From equation (9), it can be seen that $F_{l}$ can be determined by the tendon force $T_{0}$, the distal segment angle $\theta_{1}$, and the lever arm $k_{1}$; also $F_{2}$ can be determined by $\mathrm{T}_{0}, \theta_{1}, \theta_{2}$, and $k_{2}$.

The contact force transmission characteristic of this UA mechanism is analyzed below. By fixing the contact points and plotting the two contact forces over all sets of angle combinations, Fig. 5 can be obtained.

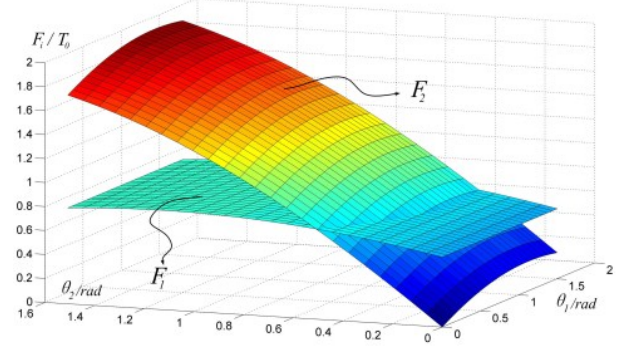

Fig. 5.The force transmission characteristic

Notes: in this figure, the contact point is fixed, $k_{l}=k_{2}=\left(L_{l}+L_{0}\right) / 2$; and all the other design parameters are the same as the specified design which will be discussed later; the range of both angles is from 0 to $\pi / 2$.

According to the results of Fig. 5, the transmission ratio $F_{i} / T_{0}$ remains relatively high as the two angles change, and in particular $F_{1} / T_{0}$ remains relatively constant during the closing stage. However, $F_{2}$ is very small when the middle segment angle is near zero, which is acceptable since the middle segment will bend during most grasp tasks.

\section{Design of Actuation System}

The Columbia Hand constitutes three fingers integrated on a palm, one of which is able to rotate around the wrist (acting as a thumb). Instead of applying three lifting mechanisms (one for each finger), there is one mechanism for all three fingers. While various methods of actuation exist, such as a planetary gear system [13], this article proposes a movable 
block mechanism driven by a single linear actuator. This allows all unconstrained fingers to move even if one finger is externally locked. The mechanism accomplishes this by creating parallel kinematic chains which allow common sets of tendon wire to be "shared" by all three fingers.

\section{A. Principle of the Movable Block mechanism}

The movable block mechanism shown in Fig. 6 was proposed to provide tendon force and vertical displacement for the three fingers. This section analyzes this system in detail, utilizing techniques similar to those described in [6].

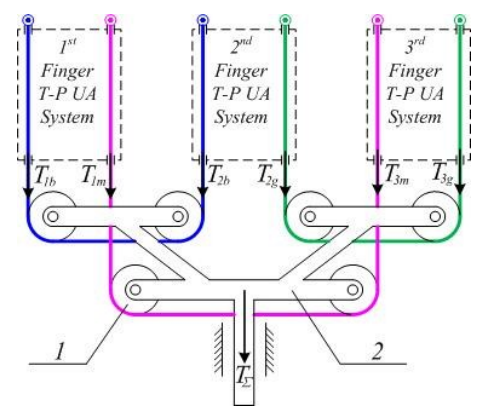

Fig. 6. The movable block mechanism, which allows all three fingers to be controlled by a single motor but still conform to an arbitrary object shape. Notes: 1 - the movable pulley; 2 - the rigid frame; $T_{k j}$ e.g. $T_{l b}-$ the tendon force produced by the blue tendon in the figure; $T_{\Sigma}-$ the force applied on the frame (produced by the lead-screw mechanism).

Though the analysis in the previous section considered one wire within each finger, the actual actuation mechanism in our design includes a pair of wires running symmetrically through each figure. Each finger has two wires attached to the distal phalanx, but each of the other two ends of these wires is attached to the distal phalanx of one of the other fingers. The wires are routed around a set of pulleys in order to minimize friction in the system. Thus, each finger is kinematically coupled with the other two fingers, and forcing one finger to extend would cause the other two fingers to close.

Fig. 6 yields the following kinematic constraints:

$$
\begin{gathered}
\Delta l_{1 b}+\Delta l_{2 b}=\Delta l_{1 m}+\Delta l_{3 m}=\Delta l_{2 g}+\Delta l_{3 g}=2 \Delta d_{\Sigma} \\
\Delta l_{1 b}-\Delta l_{1 m}=\Delta l_{2 b}-\Delta l_{2 g}=\Delta l_{3 m}-\Delta l_{3 g}=0
\end{gathered}
$$

where $d_{\Sigma}$ is the distance traveled by the linear actuator, $\Delta l_{j, k}$ is the length change of $k$ color tendon within the $j$ th finger.

Also, the following force constraints can be obtained:

$$
\begin{gathered}
T_{1 b}-T_{2 b}=T_{1 m}-T_{3 m}=T_{2 g}-T_{3 g}=0 \\
T_{1 b}+T_{2 b}+T_{1 m}+T_{3 m}+T_{2 g}+T_{3 g}=T_{\Sigma}
\end{gathered}
$$

where $T_{j, k}$ is the tension of the $k$ color wire in the $j$ th finger.

Now consider the total force exerted by one particular finger on an object during a grasp:

$$
F_{k}=\left[\begin{array}{c}
F_{k, x} \\
F_{k, y} \\
F_{k, z}
\end{array}\right]=\left[\begin{array}{lll}
\hat{n}_{1, k} & \hat{n}_{2, k} & \hat{n}_{3, k}
\end{array}\right]\left[\begin{array}{l}
\eta_{1, k} \\
\eta_{2, k} \\
\eta_{3, k}
\end{array}\right]\left(\sum_{j} T_{k, j}\right)
$$

where $\widehat{\boldsymbol{n}}_{i, k}$ is the unit normal of the $i$ th segment contact for the $k$ th finger, and $\eta_{i, k}$ is the transmission ratio $\left(F_{i} / T_{0}\right)$ of the for the ith finger contact and the $k$ th finger. Recall equation $(4,5)$; $\eta_{i, k}$ is independent of $T_{0}$. The normal and transmission ratio matrices can be combined into a single Jacobian matrix $J$, which is a function of the geometry: $\quad F_{k}=J_{k}\left(\Sigma_{j} T_{k, j}\right)$
The pseudo-inverse can then be taken of the Jacobian to solve for the sum of tension in the wires in that finger:

$$
J_{k}^{+} \boldsymbol{F}_{k}=\left(\sum_{j} T_{k, j}\right)(17), \quad\left(T_{1 b}+T_{1 m}\right)=J_{1}^{+} \boldsymbol{F}_{1}(18)
$$

Substituting these results into to equation (14),

$$
T_{\Sigma}=\sum_{\substack{j=1,2,3 \\ k=b, m, g}} T_{j, k}=\sum_{p=1,2,3} J_{p}^{+} F_{p}
$$

Consider a change in the force applied by the linear actuator,

$$
\Delta T_{\Sigma}=\sum_{p}\left\{\left(J_{p}^{+}\right) \Delta F_{p}+\left(\Delta J_{p}^{+}\right) F_{p}\right\}
$$

Assuming no change in geometry, as would be the case for a closed grasp, (20) simplifies to: $\Delta T_{\Sigma}=\sum_{p}\left(J_{p}^{+}\right) \Delta F_{p}$

This demonstrates that the force applied by the motor is distributed across the fingers according to their geometry. Particularly, with a constant force applied by the actuator, i.e. $\Delta T_{\Sigma}=0$, the external forces applied by the fingers remain coupled and thus may still change according to (21).

Thus, one benefit is that an external force applied to one finger increases the tension in that finger. Because the tensions are coupled, the disturbance on that finger will result in compensation forces applied by the other fingers. With the appropriate grasping geometry, this means that a disturbance force on a grasped object actually results in a tighter grasp.

\section{B. Design and Integration of the Actuation System}

As shown in Fig. 7, the actuation system includes the lead-screw transmission connected to the motor and the tendons routed throughout the palm. The lead-screw transmission provides a non-backdrivable characteristic, allowing for accurate positioning.

The actuation system also includes the control of thumb rotation. Here, a worm-gear mechanism is utilized to provide non-backdrivable abduction and adduction of the thumb.

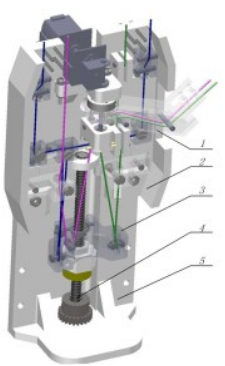

(a)

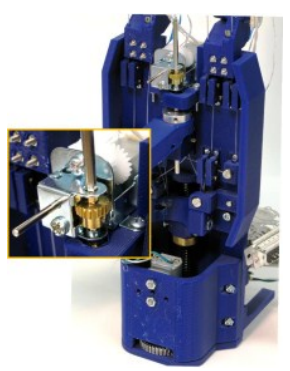

(b)
Fig. 7.Actuation system design featuring a single lead-screw mechanism for the tendons and a non-backdrivable motor for the thumb rotation. Notes: 1 - the thumb;2 - palm;3 - the lifting mechanism;4 - the lead screw transmission; 5 - the wrist frame

\section{FINGER DESIGN \& SENSOR INTEGRATION}

The Columbia Hand successfully integrates joint angle sensors and force sensors on each finger segment. Specifically, there are ten joint angle sensors (nine for finger joints and one for the thumb rotation) and nine force sensors (one for each segment).

It is important to integrate sensors into a UA hand. Angle sensors allow one to achieve position feedback and therefore gain knowledge of object shape, while force sensors allow one to obtain contact forces. These measurements can 
provide substantial information regarding object shape as well as grasp quality, which are vital for the successful manipulation of an object. Such sensory integration therefore adds the benefits of position feedback often obtained in fully actuated hands.

\section{A. The Finger Design with Sensor Integration}

As shown in Fig. 8, ball bearings are employed in each

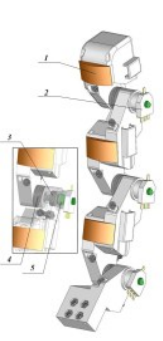

(a)

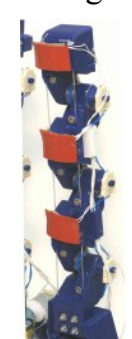

(b)
Fig. 8.Finger design with sensors Notes: 1 - force sensor; 2 - angle sensor; 3 -ball bearing; 4 - thrust bearing; 5 -hex-shaft joint. Each finger extends 153 $\mathrm{mm}$, and the design parameters defined previously are set as $L_{0}=36 \mathrm{~mm}, L_{l}=12 \mathrm{~mm}, R_{0}=14 \mathrm{~mm}$. Every joint utilizes a rotary potentiometer as an angle sensor, which has several advantages.

First, potentiometers offer a very linear characteristic and high accuracy (as demonstrated in the following section). Second, they are very compact, inexpensive, and straightforward to implement. Third, compared to Hall Effect sensors, potentiometers are smaller and will not be affected by metal components like bearings and return springs or magnetic properties of grasped objects.

Also, each segment has a convex curve equipped with a Force Sensitive Resistor (FSR), which is covered by a grip pad. While other research features capacitive tactile sensors [10] or piezoelectric polymer film elements [9], FSRs provide an inexpensive, sensitive, simple alternative. The grip pads are relatively soft and therefore conform to objects. This, in combination with a high coefficient of friction, helps distribute forces over the FSRs' entire surface area and increases the quality of grasps.

The FSR circuitry constitutes a simple voltage divider. While the FSR is a nonlinear device which can be highly sensitive to changes in low forces but much less sensitive to changes at high forces, the nonlinear transfer characteristic of the voltage divider helps compensate by inducing greater output changes at smaller values of the sensor's resistance. For the angle sensors, however, a non-inverting op amp configuration is employed with the potentiometer as the feedback resistor. This produces a linearly changing voltage in response to a linearly changing resistance.

\section{B. Sensor Performance}

Currently, the force sensors are only used for binary data (whether a segment has contacted the object); if a segment's FSR produces a voltage above a predetermined reference, it will indicate contact. This data is sufficient to substantially increase grasp quality and control (see [9]).

The angle sensors, however, must provide precise information for position feedback. They are tested as below, with ground truth obtained with a MicroScribe. In this test, one joint is set to several positions while data is recorded from the MicroScribe, voltage output, and Matlab. Fig. 9 (a) indicates a good linear characteristic of the circuitry while Fig. 9 (b) indicates a high overall position feedback accuracy.

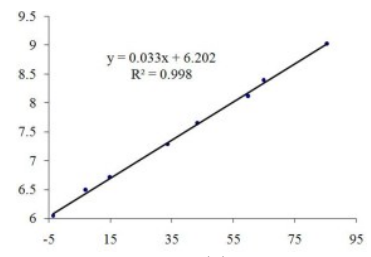

(a)

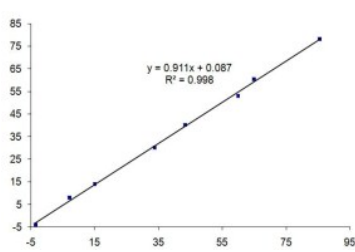

(b)
Fig. 9. The (a) voltage output and (b) angle determined by Matlab is plotted versus the actual angle. High correlation coefficients as well as a slope very close to 1 indicate the position feedback system's high degree of accuracy.

\section{TESTS}

The prototype of the Columbia Hand is shown as Fig. 10.

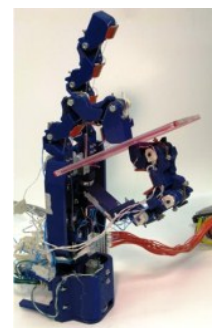

(a)

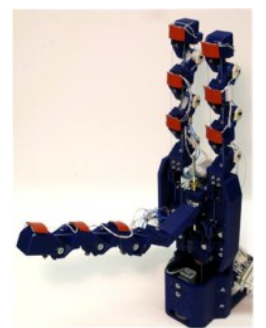

(b)
Fig. 10. The complete design of Columbia Hand, featuring angle and tactile sensors as well as a TPUA mechanism driven by a single motor.

It has three fingers, each of which has three segments, and a rotational DOF at the base of the thumb. There are two actuators, one controlling the 9 DOFs of the fingers and the other controlling the thumb's position. There are ten angle sensors and nine force sensors. This data is received by a DAQ board and processed via Simulink.

\section{A. The Pre-shaping Test}

Fig. 11 compares plots generated by Matlab based on the angle sensors in one finger as it closes without an object with pictures of the actual finger during the same test.

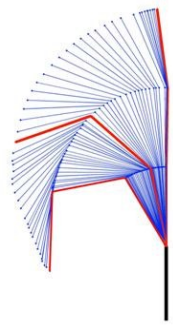

(a)

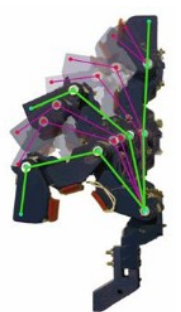

(b)

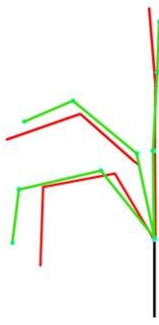

(c)
Fig. 11. Results of the pre-shaping test with an individual finger: (a)shows the animation computed from sensory feedback while (b)shows the ground truths; (c) compares the first, middle, and last positions of (a) and (b). These demonstrate a high degree of accuracy of the position feedback system and a natural preshaping process strongly resembling the prediction above.

It can be concluded from the results that i) the finger has a relatively large workspace; ii) Fig. 11 (c) indicates the accurate real-time tracking provided by the angle sensors; iii) the test results differ slightly from the predictions of the previous analysis (Fig. 3). These discrepancies can be attributed to gravity as well as friction; resistance in the joints delays the rotation of lower joints, causing the distal segment to rotate substantially before the others begin to move, creating the observed differences.

The second test is similar to the first test, but investigates 
the pre-shaping of the whole hand. As shown in Fig. 12, pictures are continuously taken and the position feedback is presented via Matlab. It indicates that the movement is very natural and stable and that the position feedback is accurate.

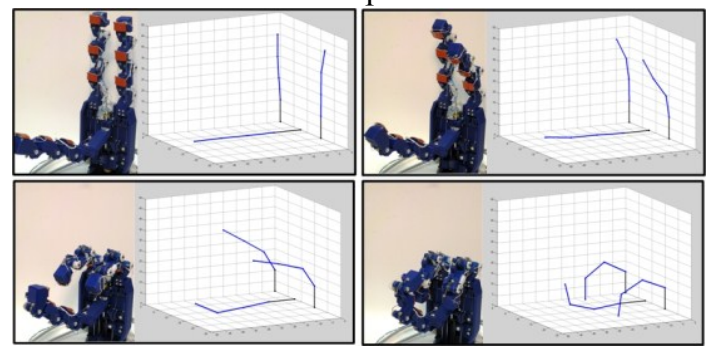

Fig. 12.The results of pre-shaping stage of the whole hand. The right-hand pictures show the animation generated by Matlab using the sensory feedback, while the left-hand pictures show the ground truths. These results demonstrate a very human-like characteristic.

\section{B. The Grasp Test}

In this section, two objects are grasped by the Columbia Hand while sensory feedback is recorded: a cylindrical CD case and a toy horse. In the animations produced by Matlab, a red segment indicates contact on that surface with the grasped object. These results are consistent with the ground truth next to each animation. The results are shown as Fig. 13.

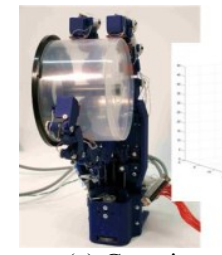

(a) Grasping a CD case

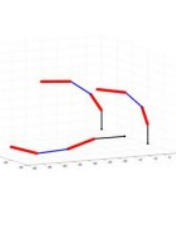

Fig. 13 The Columbia Hand successfully grasping objects. The right column

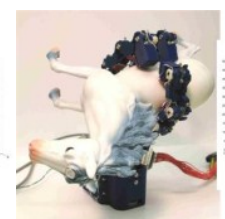

(b) Grasping a toy horse shows plots generated by Matlab from sensor data; red segments indicate contact with the object. These demonstrate stable grasps for differently shaped objects and the ability to predict grasp quality based on sensory data.

It can be concluded from the results that i) the Columbia hand can successfully adapt to different shapes; ii) the angle sensors are successfully integrated and provide precise positional information; iii) the force sensors are sensitive and work well as logic indicators for whether or not contact has been achieved. From this data, one can obtain knowledge about object shape and grasp quality before attempting to manipulate the object. Such integration therefore facilitates the operation of underactuated hands in unknown environments or situations which require stable grasps; it combines many benefits offered by full actuation with the simplicity and adaptability of underactuation.

\section{FUTURE WORK AND CONCLUSION}

There are several interesting issues which can be considered in future research. First, the pre-shaping process can be optimized by modifying the design parameters, so that the workspace of each finger and variety of graspable objects can be maximized. Second, a control law can be considered to control the grasp process with feedback information from the sensors, thereby utilizing the angle and force information to enhance grasp quality and control. Similarly, the force sensors can be used to provide analog force values rather than binary ones - this can consequently provide more thorough information regarding grasp quality as well as object properties. Benchmark tests can also be performed to facilitate comparison of this hand with other underactuated hands, as proposed in [14]. Finally, the hand will also be mounted as the end effector of a robotic arm, allowing the hand to operate as part of a larger system and interact with the surrounding environment.

This paper proposed a novel underactuated robotic hand design - the Columbia Hand. It has three fingers, which are implemented with tendon pin underactuation mechanisms. The three fingers are actuated by only two motors, one of which controls the thumb rotation. The Columbia Hand is equipped with angle and force sensors, which can provide precise angle feedback and sensitive binary force feedback. Furthermore, this style of implementation provides a system which can be analyzed theoretically to predict contact forces as well as hand position given a particular object shape. Tests indicate that the Columbia Hand is functional at grasp tasks for a variety of objects. Additionally, its practical feedback allows for grasp quality analysis and object recognition as well as improved control algorithms that can compensate for intrinsic error. Such developments demonstrate the power of underactuated hands and their growing potential for humanoid robotics.

\section{REFERENCES}

[1] A.M. Dollar and R. D. Howe, "A robust Compliant Grasper via Shape Deposition Manufacturing” IEEE/ASME transactions on mechatronics vol. 11, No. 2, April 2006

[2] J. Tegin et al., "Real life Grasping using an Under-Actuated Robot Hand - Simulation and Experiments", IEEE International Conference on Advanced Robotics, 2009, Munich, Germany

[3] 2010 IFToMM/ASME Workshop on Underactuated Grasping, Ecole Polytechnique de Montreal, Montreal Canada

[4] K. Kaneko, K. Harada, F. Kanehiro, Development of Multi-fingered Hand for Life-size Humanoid Robots, ICRA2007, pp. 913-920, 2007.

[5] Shadow Robot Company, "Design of a Dextrous hand for CLAWAR applications" 2003 International Conference on Climbing and Walking Robotics, Catania, Italy

[6] L. Birglen, T. Laliberté and C. Gosselin, Underactuated Robotic Hands, Springer, 2008.

[7] B. Bergelin, B. Slaboch, J. Sun, and P. A. Voglewede, "A handy new design paradigm”, Mechanical Sciences 2(1), pp. 59-64, 2011.

[8] P. J. Kyberd, A. Clawson, and B. Jones, "The use of underactuation in prosthetic grasping”, Mechanical Sciences 2(1), pp. 27-32, 2011.

[9] Dollar, A.M., Jentoft, L.P., Gao, J.H., Howe, R.D.,"Contact sensing and grasping performance of compliant hands", Autonomous Robots 28(1), 65-75 (2010)

[10] J. S. Son, M. R. Cutkosky, and R. D. Howe, "Comparison of contact sensor localization abilities during manipulation," Robotics and Autonomous Systems, 17(4):217-233, June 1996.

[11] L. Birglen, "From flapping wings to underactuated fingers and beyond: a broad look to self-adaptive mechanisms", Mechanical Sciences 2(1), pp. 5-10, 2011

[12] L. Birglen, "The kinematic preshaping of triggered self-adaptive linkage-driven robotic fingers", Mechanical Sciences 2(1), pp. 41-49, 2011.

[13] K. Koganezawa, and Y. Ishizuka, Novel Mechanism of Artificial Finger Using Double Planetary Gear System, IROS2008, pp. 3184-3191, 2008.

[14] G. A. Kragten, C. Meijneke, and J. L. Herder, "A proposal for benchmark tests for underactuated or compliant hands", Mechanical Sciences 2(1), pp. 13-18, 2011. 